\title{
Neisseria gonorrhoeae Pilus Attenuates Cytokine Response of Human Fallopian Tube Explants
}

\author{
Luis Velasquez, ${ }^{1}$ Katherine García, ${ }^{1}$ Francisco Morales, ${ }^{1}$ John E. Heckels, ${ }^{2}$ Pedro Orihuela, ${ }^{1}$ \\ Paula I. Rodas, ${ }^{1}$ Myron Christodoulides, ${ }^{2}$ and Hugo Cardenas ${ }^{1}$
}

${ }^{1}$ Facultad de Quimica y Biología, Universidad de Santiago de Chile, Casilla 40 Correo 33, 9170022 Santiago, Chile
${ }^{2}$ Neisseria Research Group, Sir Henry Wellcome Laboratories, Division of Infection, Inflammation and Immunity,
University of Southampton Medical School, Southampton SO16 6YD, UK

Correspondence should be addressed to Hugo Cardenas, hugo.cardenas@usach.cl

Received 4 July 2011; Revised 10 October 2011; Accepted 16 October 2011

Academic Editor: Lori Snyder

Copyright (c) 2012 Luis Velasquez et al. This is an open access article distributed under the Creative Commons Attribution License, which permits unrestricted use, distribution, and reproduction in any medium, provided the original work is properly cited.

\begin{abstract}
Background. A role for pilus during attachment of Neisseria gonorrhoeae to epithelia of the female reproductive tract is currently assumed. However, $\mathrm{Pil}^{-}$gonococci have been observed during infection of the reproductive tract, which prompted us to examine the effect of pili on the dynamics of infection and the inflammatory responses of mucosal explants of the human Fallopian tube. Methods. Mucosal explants were infected in vitro with Opa negative $\mathrm{Pil}^{-}$and $\mathrm{Pil}^{+} \mathrm{N}$. gonorrhoeae strains. Results. Piliation enhanced gonococcal adherence to the epithelium within $3 \mathrm{~h}$ of infection $(P<0.05)$ but thereafter did not offer advantage to gonococci to colonize the epithelial cell surface $(P>0.05)$. No differences were found between the strains in numbers of gonococci inside epithelial cells. Pil ${ }^{-}$bacteria induced higher levels $(P<0.05)$ of IL- $1 \beta$, TNF- $\alpha$, GM-CSF, MCP-1, and MIP- $1 \beta$ than Pil ${ }^{+}$bacteria. There were no differences between both strains in LOS pattern, and Pil expression did not change after coincubation with mucosal strips. Conclusions. Results show that gonococcal invasion of the human Fallopian tube can occur independently of pilus or Opa expression, and suggest that pilus, by inhibition of several key elements of the initial inflammatory response, facilitates sustained infection of this organ.
\end{abstract}

\section{Introduction}

Neisseria gonorrhoeae (NGO) is an exclusive pathogen of humans that infects the mucosal epithelia of the male urethra and the female lower genital tract. It is the etiological agent of gonorrhea, and, for women in particular, infection can spread into the upper reproductive tract, including the Fallopian tubes (FTs). Gonococcal interactions with the FT epithelium initiate salpingitis, an inflammatory response that can lead to FT damage and infertility. In the FT, evidence from the classical papers of McGee and colleagues using a culture model of ex vivo FT organ explants suggested that gonococcal interactions were mediated by expression of type IV pilus and the phase-variable colony opacity (Opa) protein adhesins $[1,2]$. Moreover, evidence gathered with many isolated human cell lines has also shown that invasion of host cells by gonococci involves initial attachment mediated primarily by pili [3-9] which leads to intracellular cortical plaque formation [10] with cooperation with Opa proteins leading to bacterial internalization and/or traversal of epithelial barriers [11-15].

In the course of research exploring the influence of contraceptives on gonococcal infection of the human FT [16], we used a clinical isolate that afterwards was phenotypically characterized as a $\mathrm{Pil}^{-} \mathrm{Opa}^{+}$strain [17]. Attachment and internalization of this particular strain into the oviductal epithelium were observed from the first minutes of exposure and were followed by the release of proinflammatory cytokines such as IL- $1 \alpha$, IL- $1 \beta$, and TNF- $\alpha$ [17]. It has been suggested that in addition to mediating bacterial interactions, pili also have a role in innate immunostimulation by influencing the cytokine response induced during infection [18]. In the current study, we examined the correlation between pilus expression in gonococcal variants lacking Opa protein on bacterial interactions with FT mucosal explants and the induction of cytokine secretion. 


\section{Materials and Methods}

2.1. Neisseria gonorrhoeae Strains. Neisseria gonorrhoeae (NGO) strains $\mathrm{P} 9$, variant $1\left(\mathrm{Pil}^{-} \mathrm{Opa}^{-}\right)$, and variant 2 $\left(\mathrm{Pil}^{+} \mathrm{Opa}^{-}\right)$[19] were grown on $\mathrm{GC}$ agar at $37^{\circ} \mathrm{C}$ in $5 \%$ $(\mathrm{v} / \mathrm{v}) \mathrm{CO}_{2}$. The strains were derived from the original P9 isolate $[20,21]$, and the strain phenotype was confirmed by examining colony morphology with a stereomicroscope and with SDS-PAGE analysis and immunoblotting with specific monoclonal antipilus antibody SM1 [22] and anti-Opa protein antibody B33 [14], both before and after coincubation with tubal mucosal explants.

\subsection{Miniscale LOS Extraction and Tricine-SDS-PAGE Analisis.} To verify LOS integrity in the two NGO strains, LOS samples were extracted and visualized by subjecting proteinase $\mathrm{K}$ digests of whole-cell lysates to Tricine SDS-PAGE [23, 24]. After electrophoresis, the gel was fixed in a solution containing 40\% ethanol and 5\% acetic acid overnight followed by silver staining [25].

2.3. Human Fallopian Tube Explants. Fallopian tubes (FTs) were obtained after informed consent from 13 fertile donors submitted to gynecological surgery for reasons unrelated to this study. Exclusion criteria were sexually transmitted disease during the last year and no history of pelvic inflammatory disease. Protocols were approved by the Ethics Committees of the Universidad de Santiago de Chile and the Hospital San Jose (Santiago, Chile) where the surgeries were carried out. Organs were processed immediately after removal as previously described [16]. Briefly, after muscle dissection, mucosal strips were cut into $1 \mathrm{~cm}^{3}$ segments and cultured in Dulbecco's modified Eagle's medium (DMEM, Hi Clone) for $3 \mathrm{~h}$ before infection with NGO.

2.4. Infection of Human FT Explants with NGO. Mucosal explants were infected with $100 \mu \mathrm{L}$ of NGO suspension $(3 \times$ $10^{6} \mathrm{cfu} / \mathrm{mL}$ ) in DMEM as previously described [17]. Adherent and internalized gonococci in the mucosal strips were examined under confocal microscopy (Axiovert 100M Zeiss laser scanning microscope) and counted as described previously [16]. Briefly, tissues for microscopy were fixed in cold 4\% (v/v) paraformaldehyde in PBS, pH 7.4 (Winkler, Santiago, Chile) for $1 \mathrm{~h}$ before transfer to $10 \%(\mathrm{w} / \mathrm{v})(1 \mathrm{~h})$ and $30 \%$ $(\mathrm{w} / \mathrm{v})$ (overnight) sucrose. They were mounted in embedding compound (Cryo-M-Bed, Bright Instruments Co. Ltd., Huntingdon, UK) and frozen at $-20^{\circ} \mathrm{C}$. Sections of $6 \mu \mathrm{m}$ were cut using a Bright Starlet Cryostat, and gonococci were stained with a rabbit antigonococcal antiserum and fluorescein-conjugated anti-rabbit IgG.

2.5. Cytokine Analysis. Samples from the culture media were taken at intervals up to $24 \mathrm{~h}$ of coincubation and the secretion of cytokines IL- $1 \beta$, IL-6, TNF- $\alpha$, IL-10, TGF- $\beta$, IL- 8 , GM-CSF, MCP-1, MIP- $1 \beta$, and RANTES was quantified by sandwich immunoassays as described previously [26].

2.6. Statistical Analysis. Statistical analysis was done by ANOVA or $t$-test as required, using Minitab Software
TABLE 1: Attachment of NGO variants to human FT explant epithelium. FT explants were infected with $\mathrm{Pil}^{-} \mathrm{Opa}^{-} \mathrm{NGO}(n=7)$ and $\mathrm{Pil}^{+} \mathrm{Opa}^{-}(n=6)$, and 16 fields were examined for each explant at each time point. In each field, gonococci were counted within a continuous epithelial strip of at least 150 cells. The data are presented as the mean and SEM of the number of gonococci observed, attached, or internalized. * denotes a significantly $(P<$ 0.05 ) higher number of attached $\mathrm{Pil}^{+} \mathrm{Opa}^{-} \mathrm{NGO}$ compared to the $\mathrm{Pil}^{-} \mathrm{Opa}^{-}$variant at $3 \mathrm{~h}$.

\begin{tabular}{lcc}
\hline Time (h) & \multicolumn{2}{c}{ Attached } \\
\hline 3 & $2.6 \pm 0.6$ & $\mathrm{Pil}^{+} \mathrm{Opa}^{-}$ \\
6 & $4.1 \pm 1.1$ & $7.6 \pm 2.6^{*}$ \\
12 & $4.2 \pm 1.1$ & $6.3 \pm 1.4$ \\
24 & $6.8 \pm 0.9$ & $5.8 \pm 1.3$ \\
\hline \multicolumn{3}{c}{ Internalised } \\
& $\mathrm{Pil}^{-} \mathrm{Opa}^{-}$ & $6.8 \pm 2.0$ \\
\hline 3 & $12.4 \pm 1.6$ & $\mathrm{Pil}^{+} \mathrm{Opa}^{-}$ \\
6 & $14.4 \pm 1.9$ & $18.0 \pm 3.4$ \\
12 & $14.1 \pm 1.8$ & $16.8 \pm 2.4$ \\
24 & $25.1 \pm 1.9$ & $15.0 \pm 2.4$ \\
\hline
\end{tabular}

(Minitab 15.1.30.0). Comparison of attached and internalized gonococci between both strains was done by $t$-test at each incubation time using log-transformed data to correct for variance heterogeneity.

\section{Results}

Human FT explants were infected with $\mathrm{Pil}^{-} \mathrm{Opa}^{-}$and $\mathrm{Pil}^{+} \mathrm{Opa}^{-} \mathrm{NGO}$ variants, and the numbers of adherent and internalized bacteria were quantified up to $24 \mathrm{~h}$. NGO was found attached to the epithelium and internalized at all time points examined (Table 1 ), with $\mathrm{Pil}^{+}$bacteria showing a significantly higher rate of initial adherence by $3 \mathrm{~h}$ than the $\mathrm{Pil}^{-}$ variant $(P<0.05)$. Thereafter, however, no statistically significant differences were observed between the numbers of attached $\mathrm{Pil}^{+}$and $\mathrm{Pil}^{-} \mathrm{NGO}(P>0.05)$. NGO invasion of the FT epithelium was rapid, but there were no statistically significant differences $(P>0.05)$ in the numbers of $\mathrm{Pil}^{+}$ and $\mathrm{Pil}^{-}$bacteria counted within the epithelium at all time points examined (Table 1). Adherence and invasion of the FT epithelium were confirmed by confocal microscopy.

Compared to uninfected FT explants, gonococcal infection resulted in the significant $(P<0.05)$ secretion of key proinflammatory (IL-1 $\beta$, IL-6, and TNF $\alpha$ ), chemokine (MCP-1, MIP-1 $\beta$ ), and growth-factor related (MCP-1, MIP$1 \beta$ and GM-CSF) cytokines. By contrast, the secretion of IL8 , RANTES, TGF- $\beta$, MIP- $1 \alpha$, or IL- 10 was not significantly increased by gonococcal infection compared to uninfected explants (data not shown).

For those cytokines whose secretion was significantly increased by gonococcal infection compared to uninfected FT explants, secretion was influenced by pilus expression. In general, infection with $\mathrm{Pil}^{-} \mathrm{NGO}$ resulted in significantly higher secretion levels compared with $\mathrm{Pil}^{+} \mathrm{NGO}$. The levels of IL- $1 \beta$ secreted to the culture medium increased with both 

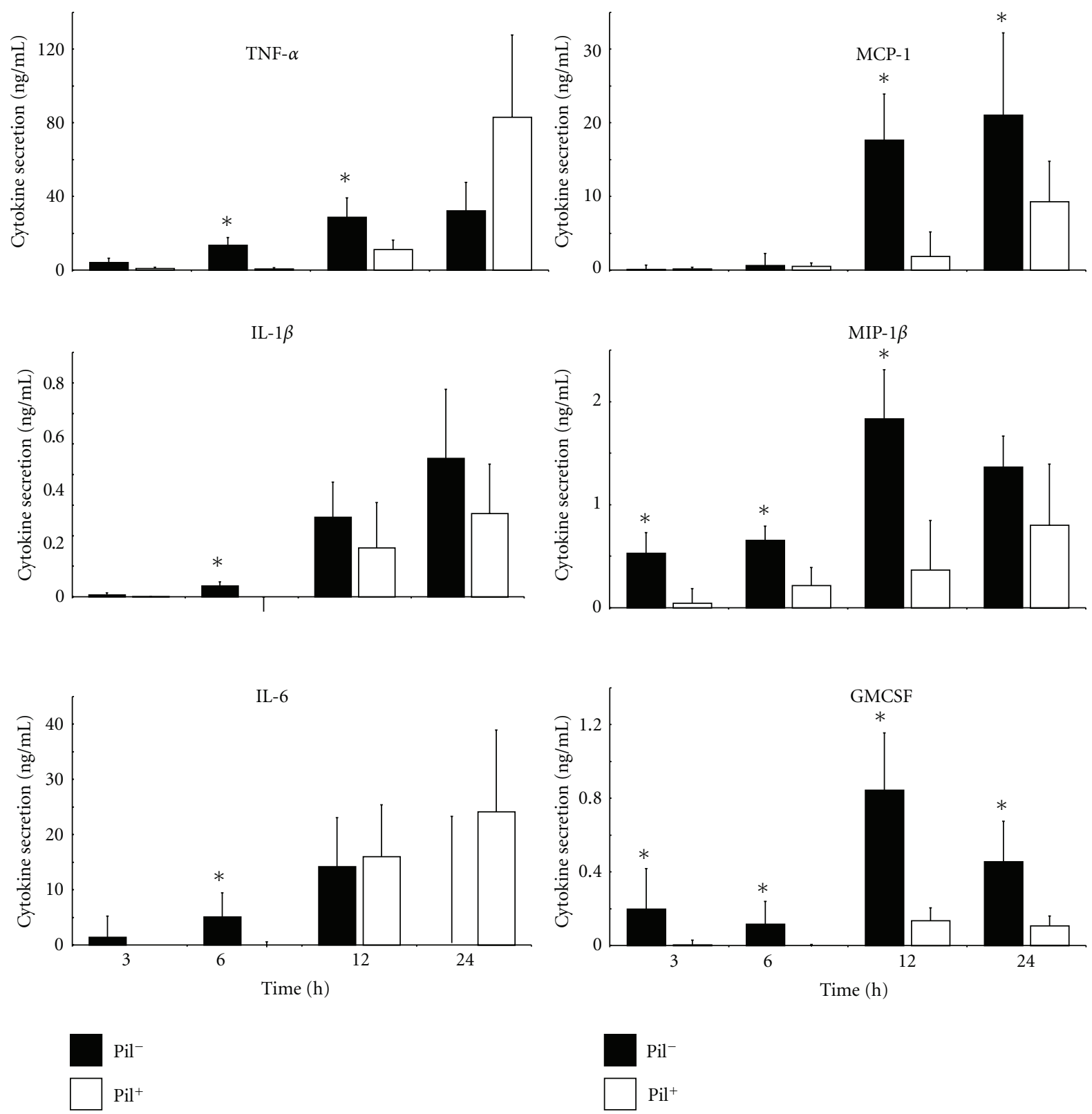

FIGURE 1: Expression of pilus attenuates cytokine production by human FT explant epithelium. FT explants were infected with Pil $^{-} \mathrm{Opa}^{-}$ NGO $(n=7)$ and $\mathrm{Pil}^{+} \mathrm{Opa}^{-}(n=6)$ and cytokine secretion measured over time. The columns represent mean cytokine levels and the standard error for the sample group. ${ }^{*}$ denotes statistically significant $(P<0.05)$ increase in cytokine secretion induced by Pil ${ }^{-}$bacteria compared to $\mathrm{Pil}^{+}$bacteria at given time point.

strains of NGO over time, but by $24 \mathrm{~h}$ they were significantly higher in explants infected with $\mathrm{Pil}^{-}$bacteria compared with $\mathrm{Pil}^{+}$bacteria $(P<0.05$; Figure 1$)$. Secretion of TNF- $\alpha$ also increased over time with both NGO strains, with the levels induced by $\mathrm{Pil}^{-}$bacteria by $12 \mathrm{hr}$ significantly higher than those observed with $\mathrm{Pil}^{+}$bacteria $(P=0.006)$, although this difference disappeared by $24 \mathrm{~h}$ (Figure 1). IL-6 secretion induced by $\mathrm{Pil}^{-} \mathrm{NGO}$ was significantly higher at the earlier time points $(3$ and $6 \mathrm{~h})$ compared with $\mathrm{Pil}^{+} \mathrm{NGO}(P<0.05)$, but these differences were not significant by $12 \mathrm{~h}$ onwards $(P>0.05)$. For MCP-1, MIP-1 $\beta$, and GM-CSF, infection with
$\mathrm{Pil}^{-}$NGO induced significantly higher amounts of secretion when compared with $\mathrm{Pil}^{+} \mathrm{NGO}$ at all time points examined $(P<0.05$; Figure 1$)$.

It is known that gonococcal phase and antigenic variation occur during in vitro growth [27] as well as during experimental infection [28], so phenotypic stability of the $\mathrm{Pil}^{-} \mathrm{Opa}^{-}$and $\mathrm{Pil}^{+} \mathrm{Opa}{ }^{-}$strains used in the current study was examined during infection of FT explants. Western blotting for pilus and Opa protein expression of NGO recovered from the FT explants demonstrated no changes in phenotype during infection (Figure 2). There were no differences in LOS 


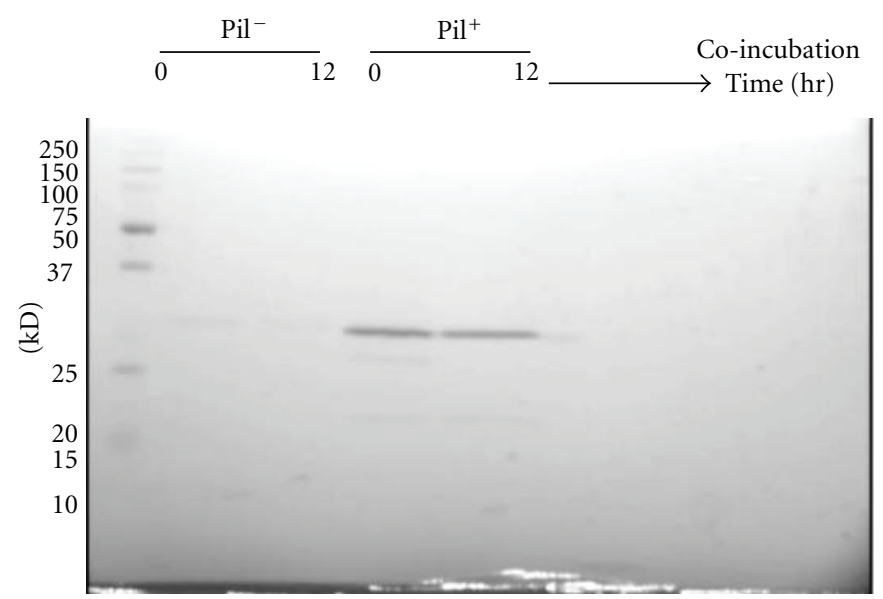

(a)

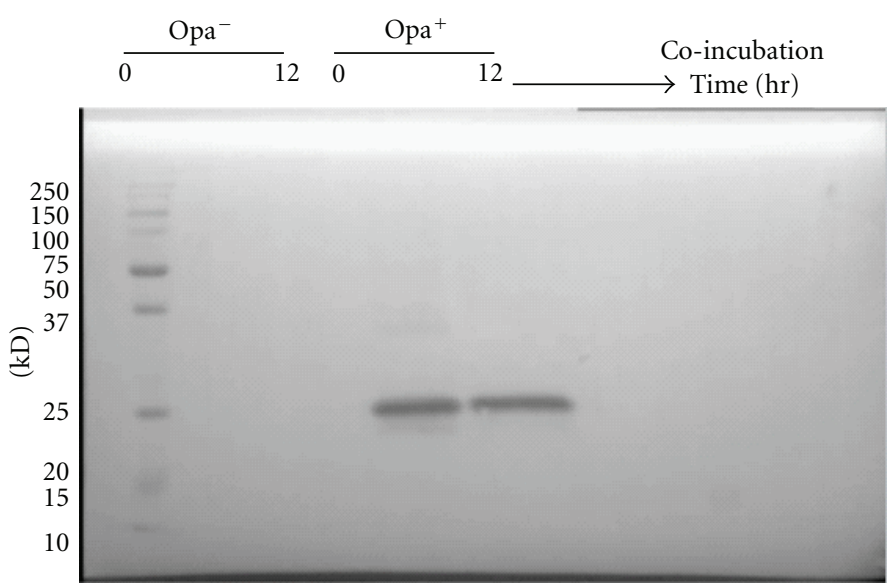

(b)

Figure 2: Western blot for Pil and Opa proteins in $\mathrm{Pil}^{+} \mathrm{Opa}^{+}$and $\mathrm{Pil}^{-} \mathrm{Opa}^{-}$gonococcal strains incubated for $12 \mathrm{~h}$ with Fallopian tube mucosal strips. (a): Western blot for Pil protein. (b): Western blot for Opa protein. The two dark bands corresponding to Pil (a) and Opa (b) appear in lanes from the $\mathrm{Pil}^{+} \mathrm{Opa}^{+}$strain only. Densitometry analysis showed no change in Pil and Opa expression after the co-incubation.

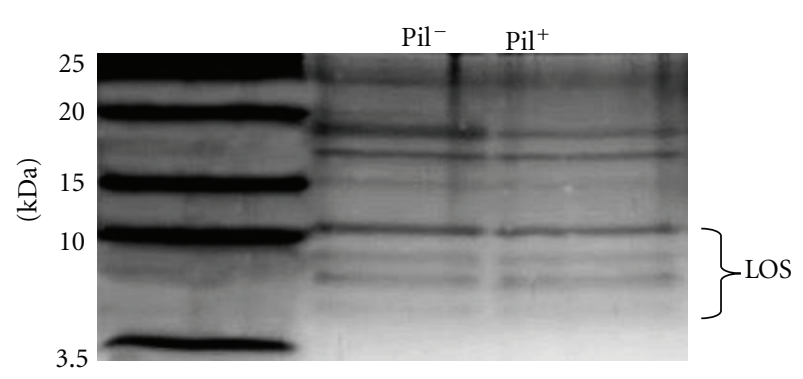

FIGURE 3: Lipooligosaccharide profiles of NGO strains used in this study. Whole-cell lysates of gonococcal strains were treated with proteinase K overnight and then boiled prior to Tricine SDS-PAGE $(16 \%)$. Protein molecular weight marker: Novex Sharp Standard (Invitrogen).

expression between both NGO strains (Figure 3). Therefore the patterns of adhesion/internalization and cytokine production in these experiments can safely be correlated with the presence or absence of pili.

\section{Discussion}

In the current study, we report an experimental in vitro examination of the role of gonococcal pili during epithelial invasion and cytokine production at the initial stages of infection of the human Fallopian tube. Experimental infection based on cell lines and cultures has provided a theoretical model for gonococcal infection in which pili have a prominent role in the initial attachment to the host cell $[18,29]$. We observed in our study that the presence of pili enhanced gonococcal adherence to the tubal epithelium within $3 \mathrm{~h}$ of infection compared with the corresponding nonpiliated variant but thereafter did not appear to offer a significant advantage to gonococci to colonize the epithelial cell surface. We have shown previously that a clinical $\mathrm{Pil}^{-} \mathrm{Opa}^{+}$gonococcal strain attached and invaded the epithelium of tubal explants [17] and the appearance of $\mathrm{Pil}^{-}$bacteria during experimental infection has also been reported [28]. In our study, pilus expression also did not confer any advantage to the gonococcus for invasion. Moreover, because the strains we used in our experiments were both Opa 
negative, our data also suggested an Opa-independent mechanism for invasion of the tubal epithelium. The redundancy of Opa is consistent with reports demonstrating that $\mathrm{Opa}^{-}$ variants were as infective as $\mathrm{Opa}^{+}$gonococci in human experimental infections [30], and adhesion and phagocytosis of $\mathrm{Pil}^{-} \mathrm{Opa}^{-}$gonococcal strains by human dendritic cells were influenced by differences in their lipooligosaccharides [31].

Taken together with the reported literature, our findings therefore suggest that mechanisms other than pilus or Opamediated binding to surface molecules of the epithelial cells operate with high efficiency during gonococci invasion of the Fallopian tube. The exact nature of these mechanisms is unclear, although it has been reported, using recombinant $E$. coli expressing specific gonococcal components, that invasion of FT epithelia depended on the expression of combinations of Opa, porin, and lipooligosaccharide [32]. However, it is also likely that the endocytic properties of the oviductal epithelium [33] probably contribute to uptake of gonococci.

In the current study, expression of pili appeared to have a role in the regulation of cytokine responses following gonococcal infection. Although both $\mathrm{Opa}^{-}$gonococcal strains induced secretion of IL- $1 \beta$, TNF- $\alpha$, GM-CSF, MCP-1, and MIP- $1 \beta$, the stimulatory effects were significantly higher with the $\mathrm{Pil}^{-}$bacteria compared to $\mathrm{Pil}^{+}$bacteria. Thus, expression of gonococcal pili appeared to attenuate cytokinechemokine responses in the mucosal epithelial cells of the human Fallopian tube, although the contribution of other uncharacterized genetic differences cannot be categorically ruled out. Or, alternatively, the absence of the large numbers of surface-located pili structures results in remodeling of the $\mathrm{OM}$ of the gonococcus to allow increased interactions with the epithelial cell LOS receptors, leading to increased cytokine production. These findings are in contrast to other studies that have investigated the involvement of pilus expression in determining the magnitude of the cytokine response. For example, during infection of a human monocytic cell line with Salmonella typhi, $\mathrm{Pil}^{+}$bacteria induced higher levels of IL- 6 production and NF- $\kappa \mathrm{B}$ activation than nonpiliated bacteria [34]. However, we should stress that the cytokine pattern observed in our experiments might not necessarily represent the in situ infection, because in vitro mucosal explants are devoid of blood and/or tissue-associated immune effector cells [35], whose contribution to the local cytokine pool is to be expected during the advanced stages of salpingitis when bacteria reach deep into the subepithelial tissue.

By contrast to pilus expression, the presence of Opa protein was not a contributory factor to cytokine attenuation in the FT epithelium itself. However, it has been shown with human dendritic cells that Opa proteins can modulate innate and adaptive immune responses, by directly suppressing Tcell receptor signaling through engagement of coinhibitory CD66a/CEACAM1 receptor, thereby raising the threshold for $\mathrm{CD}^{+} \mathrm{T}$ cell activation and proliferation to activating stimuli $[36,37]$ and interfering with antibody production by promoting human B-cell death [38]. Thus, Opa expression may play an indirect role in inhibiting innate recognition during FT infection by targeting the function of patrolling submucosal dendritic cells.
In summary, our data suggest a modulatory role for gonococcal pili during infection of the human tubal mucosa. Expression of pilus enhances the initial rapid attachment of the gonococcus to the Fallopian tube mucosal epithelium but significantly attenuates the local production of several inflammatory mediators. Although phase variation of pilus and opa expression in the upper reproductive tract is likely to be a dynamic process during interactions with the host, it is likely that the $\mathrm{Pil}^{-}$phenotype is probably present at a lower percentage of the gonococcal population. Switching off pilus expression would not be beneficial to the gonococcus in that an excess inflammatory response contributory to LOSmediated stimulation could result. Thus, a consequence of limiting the innate response of the epithelium is to reduce immune effector cell recruitment to the site of infection. This would add to the mechanisms that could allow repeated gonococcal infections in the same individual without ever generating protective immunity.

\section{Acknowledgments}

The study was supported by DICYT of USACH, FONDECYT 1090589, and Proyecto BASAL FB 0807. The authors acknowledge the skillful technical support of Mrs. Nieves Aguirre.

\section{References}

[1] Z. A. McGee, A. P. Johnson, and D. Taylor Robinson, "Human fallopian tubes in organ culture: preparation, maintenance, and quantitation of damage by pathogenic microorganisms," Infection and Immunity, vol. 13, no. 2, pp. 608-618, 1976.

[2] Z. A. McGee, A. P. Johnson, and D. Taylor-Robinson, "Pathogenic mechanisms of Neisseria gonorrhoeae: observations on damage to human fallopian tubes in organ culture by gonogocci of colony type 1 or type 4," Journal of Infectious Diseases, vol. 143, pp. 413-422, 1981.

[3] J. Swanson, "Studies on gonococcus infection. IV. Pili: their role in attachment of gonococci to tissue culture cells," Journal of Experimental Medicine, vol. 137, no. 3, pp. 571-589, 1973.

[4] W. A. Pearce and T. M. Buchanan, "Attachment role of gonococcal pili. Optimum conditions and quantitation of adherence of isolated pili to human cells in vitro," Journal of Clinical Investigation, vol. 61, no. 4, pp. 931-943, 1978.

[5] I. M. Mosleh, H. J. Boxberger, M. J. Sessler, and T. F. Meyer, "Experimental infection of native human ureteral tissue with Neisseria gonorrhoeae adhesion, invasion, intracellular fate, exocytosis, and passage through a stratified epithelium," Infection and Immunity, vol. 65, no. 8, pp. 3391-3398, 1997.

[6] M. Virji, H. Kayhty, D. J. P. Ferguson, C. Alexandrescu, J. E. Heckels, and E. R. Moxon, "The role of pili in the interactions of pathogenic Neisseria with cultured human endothelial cells," Molecular Microbiology, vol. 5, no. 8, pp. 1831-1841, 1991.

[7] H. Kallstrom, M. K. Liszewski, J. P. Atkinson, and A. B. Jonsson, "Membrane cofactor protein (MCP or CD46) is a cellular pilus receptor for pathogenic Neisseria," Molecular Microbiology, vol. 25, no. 4, pp. 639-647, 1997.

[8] T. Rudel, J. P. M. van Putten, C. P. Gibbs, R. Haas, and T. F. Meyer, "Interaction of two variable proteins (PilE and 
PilC) required for pilus-mediated adherence of Neisseria gonorrhoeae to human epithelial cells," Molecular Microbiology, vol. 6, no. 22, pp. 3439-3450, 1992.

[9] M. Kirchner, D. Heuer, and T. F. Meyer, "CD46-independent binding of neisserial type IV pili and the major pilus adhesin, PilC, to human epithelial cells," Infection and Immunity, vol. 73, no. 5, pp. 3072-3082, 2005.

[10] A. J. Merz, C. A. Enns, and M. So, "Type IV pili of pathogenic Neisseriae elicit cortical plaque formation in epithelial cells," Molecular Microbiology, vol. 32, no. 6, pp. 1316-1332, 1999.

[11] J. F. L. Weel, C. T. P. Hopman, and J. P. M. van Putten, "In situ expression and localization of Neisseria gonorrhoeae opacity proteins in infected epithelial cells: apparent role of Opa proteins in cellular invasion," Journal of Experimental Medicine, vol. 173, no. 6, pp. 1395-1405, 1991.

[12] A. J. Merz, D. B. Rifenbery, C. G. Arvidson, and M. So, "Traversal of a polarized epithelium by pathogenic Neisseriae: facilitation by type IV pili and maintenance of epithelial barrier function," Molecular Medicine, vol. 2, no. 6, pp. 745754, 1996.

[13] J. Wang, S. D. Gray-Owen, A. Knorre, T. F. Meyer, and C. Dehio, "Opa binding to cellular CD66 receptors mediates the transcellular traversal of Neisseria gonorrhoeae across polarized T84 epithelial cell monolayers," Molecular Microbiology, vol. 30, no. 3, pp. 657-671, 1998.

[14] J. M. Griffiss, C. J. Lammel, J. Wang, N. P. Dekker, and G. F. Brooks, "Neisseria gonorrhoeae coordinately uses pili and Opa to activate HEC-1- B cell microvilli, which causes engulfment of the gonococci," Infection and Immunity, vol. 67, no. 7, pp. 3469-3480, 1999.

[15] A. K. Criss and H. S. Seifert, "Gonococci exit apically and basally from polarized epithelial cells and exhibit dynamic changes in type IV pili," Cellular Microbiology, vol. 8, no. 9, pp. 1430-1443, 2006.

[16] R. Fernandez, P. Nelson, J. Delgado et al., "Increased adhesiveness and internalization of Neisseria gonorrhoeae and changes in the expression of epithelial gonococcal receptors in the fallopian tube of copper T and Norplant(R) users," Human Reproduction, vol. 16, no. 3, pp. 463-468, 2001.

[17] K. Maisey, G. Nardocci, M. Imarai et al., "Expression of proinflammatory cytokines and receptors by human fallopian tubes in organ culture following challenge with Neisseria gonorrhoeae," Infection and Immunity, vol. 71, no. 1, pp. 527-532, 2003.

[18] L. Craig, M. E. Pique, and J. A. Tainer, "Type IV pilus structure and bacterial pathogenicity," Nature Reviews Microbiology, vol. 2, no. 5, pp. 363-378, 2004.

[19] M. Virji and J. E. Heckels, "The effect of protein II and Pili on the interaction of Neisseria gonorrhoeae with human polymorphonuclear leucocytes," Journal of General Microbiology, vol. 132, no. 2, pp. 503-512, 1986.

[20] P. R. Lambden, J. E. Heckels, L. T. James, and P. J. Watt, "Variations in surface protein composition associated with virulence properties in opacity types of Neisseria gonorrhoeae," Journal of General Microbiology, vol. 114, no. 2, pp. 305-312, 1979.

[21] P. R. Lambden, J. N. Robertson, and P. J. Watt, "Biological properties of two distinct pilus types produced by isogenic variants of Neisseria gonorrhoeae P9," Journal of Bacteriology, vol. 141, no. 1, pp. 393-396, 1980.

[22] M. Virji, J. E. Heckels, W. J. Potts, C. A. Hart, and J. R. Saunders, "Identification of epitopes recognized by monoclonal antibodies SM1 and SM2 which react with all pili of Neisseria gonorrhoeae but which differentiate between two structural classes of pili expressed by Neisseria meningitidis and the distribution of their encoding sequences in the genomes of Neisseria spp," Journal of General Microbiology, vol. 135, no. 12, pp. 3239-3251, 1989.

[23] Y. L. Tzeng, A. Datta, V. Kumar Kolli, R. W. Carlson, and D. S. Stephens, "Endotoxin of Neisseria meningitidis composed only of intact lipid A: inactivation of the meningococcal 3-deoxy-D-manno-octulosonic acid transferase," Journal of Bacteriology, vol. 184, no. 9, pp. 2379-2388, 2002.

[24] H. Schägger, "Tricine-SDS-PAGE," Nature Protocols, vol. 1, pp. 16-22, 2006.

[25] C. M. Tsai and C. E. Frasch, "A sensitive silver stain for detecting lipopolysaccharides in polyacrylamide gels," Analytical Biochemistry, vol. 119, no. 1, pp. 115-119, 1982.

[26] M. Christodoulides, J. S. Everson, B. Liu et al., "Interaction of primary human endometrial cells with Neisseria gonorrhoeae expressing green fluorescent protein," Molecular Microbiology, vol. 35, no. 1, pp. 32-43, 2000.

[27] C. D. Serkin and H. S. Seifert, "Frequency of pilin antigenic variation in Neisseria gonorrhoeae," Journal of Bacteriology, vol. 180, no. 7, pp. 1955-1958, 1998.

[28] H. S. Seifert, C. J. Wright, A. E. Jerse, M. S. Cohen, and J. G. Cannon, "Multiple gonococcal pilin antigenic variants are produced during experimental human infections," Journal of Clinical Investigation, vol. 93, no. 6, pp. 2744-2749, 1994.

[29] M. Kirchner and T. F. Meyer, "The PilC adhesin of the Neisseria type IV pilus-binding specificities and new insights into the nature of the host cell receptor," Molecular Microbiology, vol. 56, no. 4, pp. 945-957, 2005.

[30] A. E. Jerse, M. S. Cohen, P. M. Drown et al., "Multiple gonococcal opacity proteins are expressed during experimental urethral infection in the male," Journal of Experimental Medicine, vol. 179, no. 3, pp. 911-920, 1994.

[31] S. J. van Vliet, L. Steeghs, S. C. M. Bruijns et al., "Variation of Neisseria gonorrhoeae lipooligosaccharide directs dendritic cell-induced T helper responses," Plos Pathogens, vol. 5, no. 10, Article ID e1000625, 2009.

[32] G. L. Gorby, A. F. Ehrhardt, M. A. Apicella, and C. Elkins, "Invasion of human fallopian tube epithelium by Escherichia coli expressing combinations of a gonococcal porin, opacityassociated protein, and chimeric lipo-oligosaccharide," Journal of Infectious Diseases, vol. 184, no. 4, pp. 460-472, 2001.

[33] C. M. Imarai, A. Rocha, C. Acuna, J. Garrido, R. Vargas, and H. Cardenas, "Endocytosis and MHC class II expression by human oviductal epithelium according to stage of the menstrual cycle," Human Reproduction, vol. 13, no. 5, pp. 11631168, 1998.

[34] F. B. Wang, X. L. Zhang, Y. Zhou, L. Ye, Z. Qi, and J. Wu, "Type IVB piliated Salmonella typhi enhance IL-6 and NF$\kappa \mathrm{B}$ production in human monocytic THP-1 cells through activation of protein kinase C," Immunobiology, vol. 210, no. 5, pp. 283-293, 2005.

[35] M. Rarick, C. McPheeters, S. Bright et al., "Evidence for cross-regulated cytokine response in human peripheral blood mononuclear cells exposed to whole gonococcal bacteria in vitro," Microbial Pathogenesis, vol. 40, no. 6, pp. 261-270, 2006.

[36] I. C. Boulton and S. D. Gray-Owen, "Neisserial binding to CEACAMI arrests the activation and proliferation of $\mathrm{CD} 4^{+} \mathrm{T}$ lymphocytes," Nature Immunology, vol. 3, no. 3, pp. 229-236, 2002.

[37] H. S. W. Lee, M. A. Ostrowski, and S. D. Gray-Owen, "CEACAM1 dynamics during Neisseria gonorrhoeae suppression of 
$\mathrm{CD}^{+} \mathrm{T}$ lymphocyte activation," Journal of Immunology, vol. 180, no. 10, pp. 6827-6835, 2008.

[38] M. Pantelic, Y. J. Kim, S. Bolland, I. Chen, J. Shively, and T. Chen, "Neisseria gonorrhoeae kills carcinoembryonic antigen-related cellular adhesion molecule 1 (CD66a)-expressing human B cells and inhibits antibody production," Infection and Immunity, vol. 73, no. 7, pp. 4171-4179, 2005. 


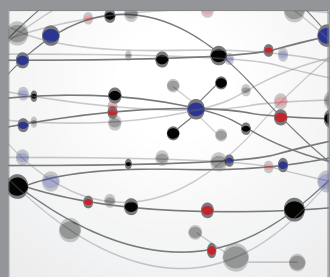

The Scientific World Journal
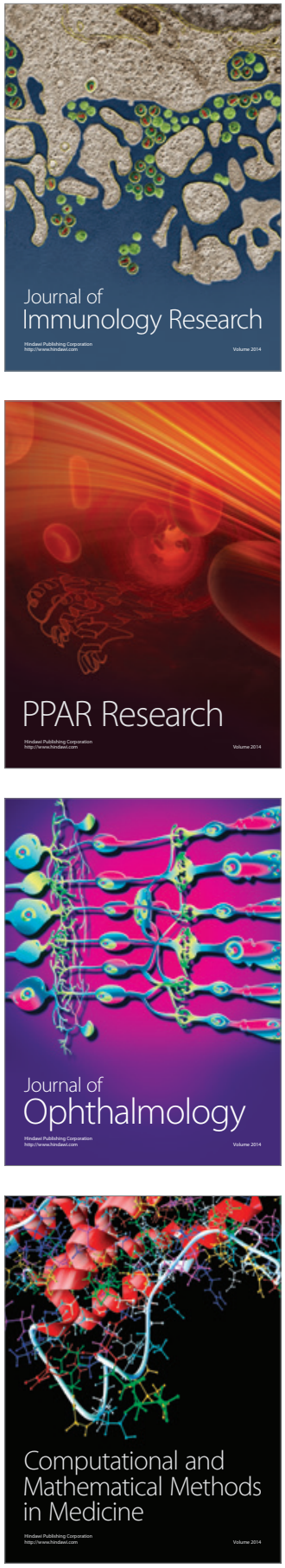

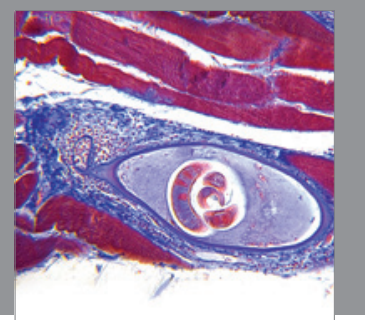

Gastroenterology

Research and Practice
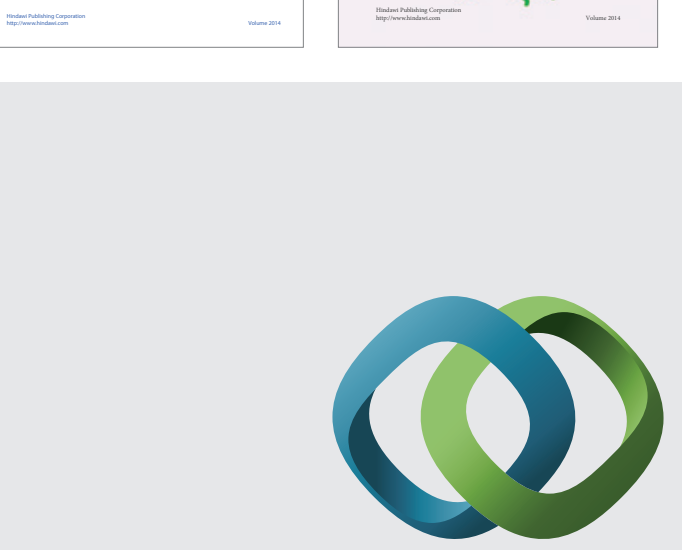

\section{Hindawi}

Submit your manuscripts at

http://www.hindawi.com
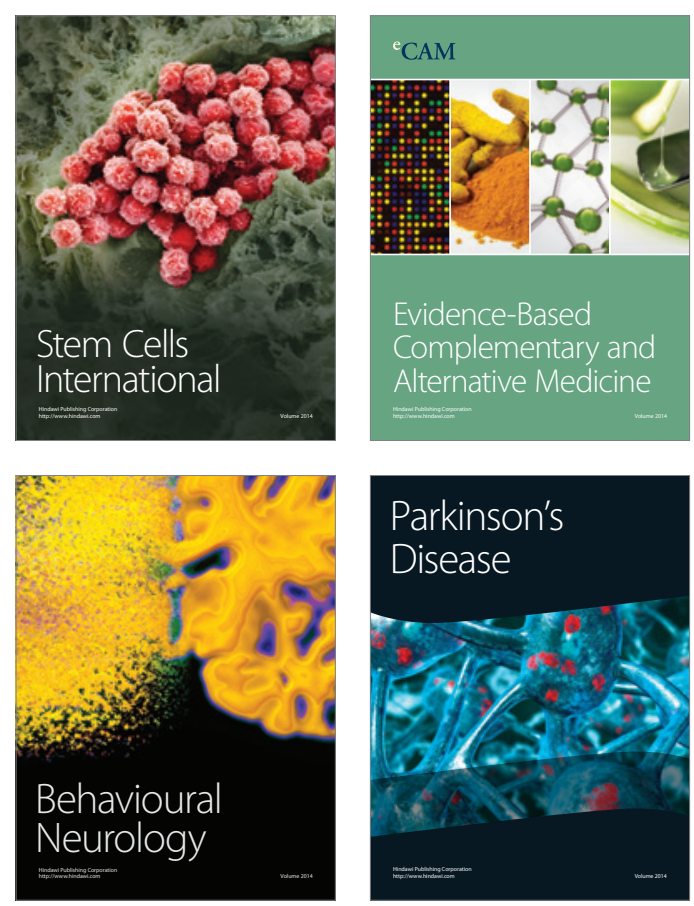

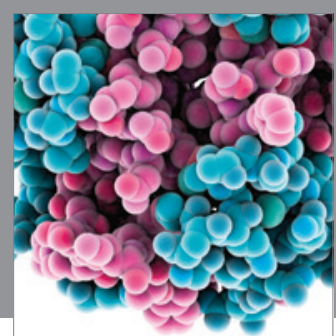

Journal of
Diabetes Research

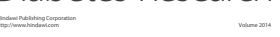

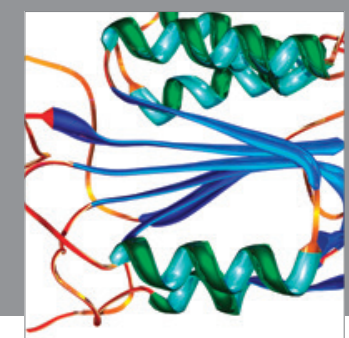

Disease Markers
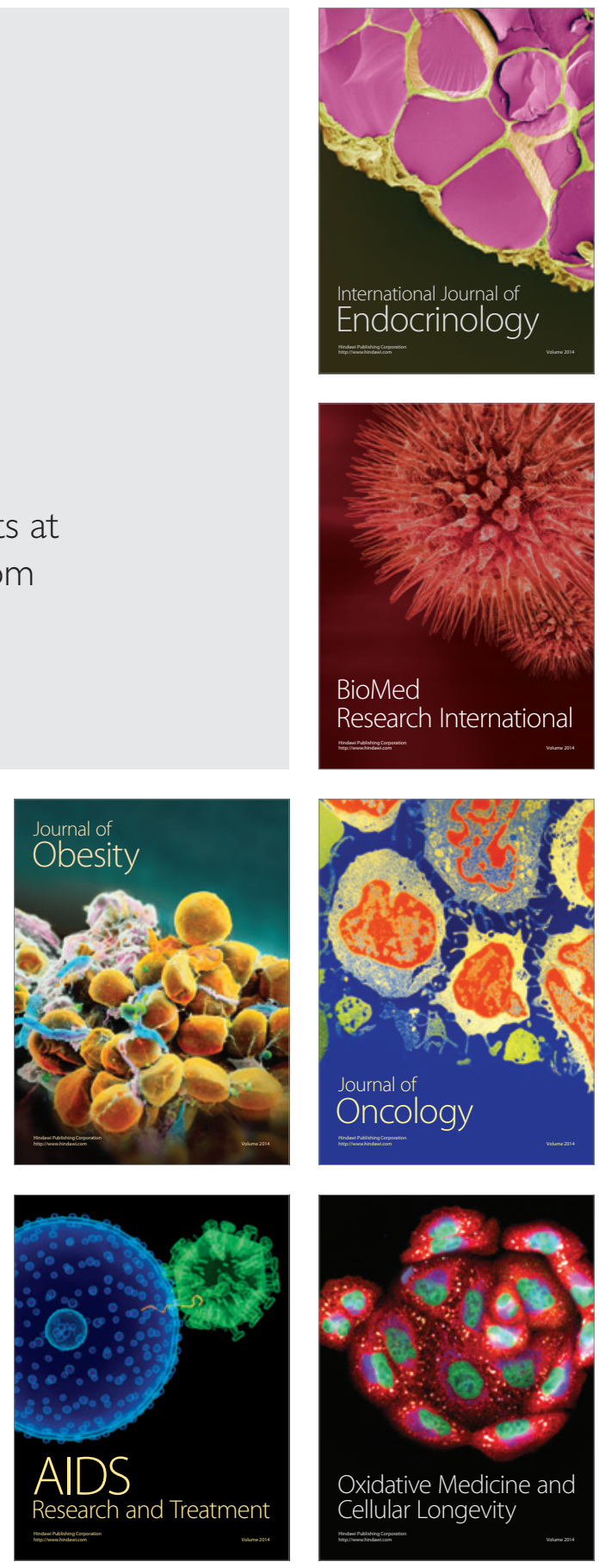